\title{
Lineal Megalithic Rock Scripts as precursors of Iberian and other lineal Mediterranean/Euro African ancient writings: the case of Fuerteventura (Canary Islands, Spain)
}

\author{
Antonio Arnaiz-Villena*1, Marcial Medina ${ }^{2}$, Valentín Ruíz-del-Valle ${ }^{1}$, \\ Adrian López-Nares ${ }^{1}$, Jose Antonio de Vera-Lima ${ }^{3}$, Luis Mata ${ }^{4}$, \\ Luis Barrera-Gutiérrez ${ }^{1}$, José Palacio-Grüber ${ }^{1}$, Fabio Suarez-Trujillo ${ }^{1}$ \\ ${ }^{1}$ Department of Immunology, Universidad Complutense, School of Medicine, Madrid, Spain. ${ }^{2}$ Freelance \\ archaeologist, Arrecife, Lanzarote, Canary Is., Spain. ${ }^{3}$ Cabildo Fuerteventura, Puerto del Rosario, CanaryIs.,Spain. \\ ${ }^{4}$ Director Museo Arqueológico de Fuerteventura, Betancuria, CanaryIs. ,Spain. ${ }^{*}$ Corresponding author. \\ Departamento de Inmunología, Facultad de Medicina, Universidad Complutense de Madrid, Pabellón 5, planta 4. \\ Avda.Complutenses/n, 28040 Madrid,Spain.E.mails: arnaizville@hotmail.com; aarnaiz@med.ucm.es.
}

(Received 22 October 2021; Accepted 26 November 2021; Published 30 November 2021)

Abstract - Lineal Megalithic/Paleolithic Lineal signs/lines may have a variety of purposes or representations. Some authors have proposed they represent sky, planets and stars and their movements, space/time representations or others, including letters/syllables or symbols/events. Some are painted, other incised; the latter are relatively more common in Megalithic scripts. Man is "writing" or creating handmade figures on stones /rocks and other supports, which sometimes have intentionally been polished since Paleolithic times: at least 70,000 years BP (Blombos Cave, South Africa). Megalithic script is named because it is associated to megalithic structures, although not exclusively. Von Petzinger 40,000 years old "symbols" and/or writing are extended worldwide in Paleolithic caves and other rocks. Man connection was worldwide in Paleolithic times. Canary Islands incise or picketed lineal writing exists with a transcribed and translated meaning collection of signs (Ibero-Guanche or Latin inscriptions and Lybic ones). Also, other African/European/Mediterranean lineal scripts there exist and examples are given in the present paper. Fuerteventura Island contains in addition many small or bigger stones and rocks with these Paleolithic/Megalithic incised lines all over its territory. About timing in which these stones that were incised by man, we are only referring to a kind of stone crafting. However, we do not discard that they were made by man several thousand years BP. Some Paleolithic/Megalithic scripts are mixed with clear Iberian semi-syllabary signs in Fuerteventura and other Canary Islands. They may reflect the evolution of more ancient Megalithic scripts to lineal writings like those detailed in the present paper and others. Finally, writing concept should be redefined whenever more precise data and dating be available.

Keywords: Fuerteventura, Canary Islands, Iberian, Guanche, Megalithic, Paleolithic, Rock Scripts, Sitovo, Gradeshnitsa, Vinca, Berber, Tuareg, Lineal, Runes, Latin, Greek, Sahara, Blombos, Lineal A, Lineal B, Cypriot, Petzinger. 


\section{Introduction}

Prehistoric scripts and the lineal megalithic rock scripts

Common prehistoric rock signs have been recorded in caves of Americas, Europe, Africa, Asia and Oceania dated between 30,000 and 10,000 years ago, which are mainly of lineal or pictographic characters (von Petzinger 2017;

https://www.openculture.com/2019/03/40000-year-old-symbols-found-in-cavesworldwide-may-represent-the-earliest-written-language.html). This implies a wide human contact throughout the World long before than previously thought. It is also supported by rock geometrical-incised signs in Blombos and Klasier River Caves, and Howiesons Poort complex rocks or pieces found in South Africa and dated, 60,000 100,000 years BC (Henshilwood \& Dubreuil 2011; Henshilwood \& D’Errico 2011; Texier et al. 2010). Some of these latter signs are similar to those described by us on the Rock of the Dead in Tenerife, Barranco de Ruiz, San Juan de la Rambla, Canary Islands (Arnaiz-Villena et al. 2019a) or at La Palma Island (Arnaiz-Villena et al. 2020a); and also to those other incise/lineal rock scripts found at all other Canary Islands (ArnaizVillena et al. 2015; 2019b; Benito-Mateo et al. 2016; de Balbín-Behrmann et al. 2009; del Arco-Aguilar et al. 2009; González-Antón et al. 1995; 2016; Asociación sociocultural Archinife 2016).

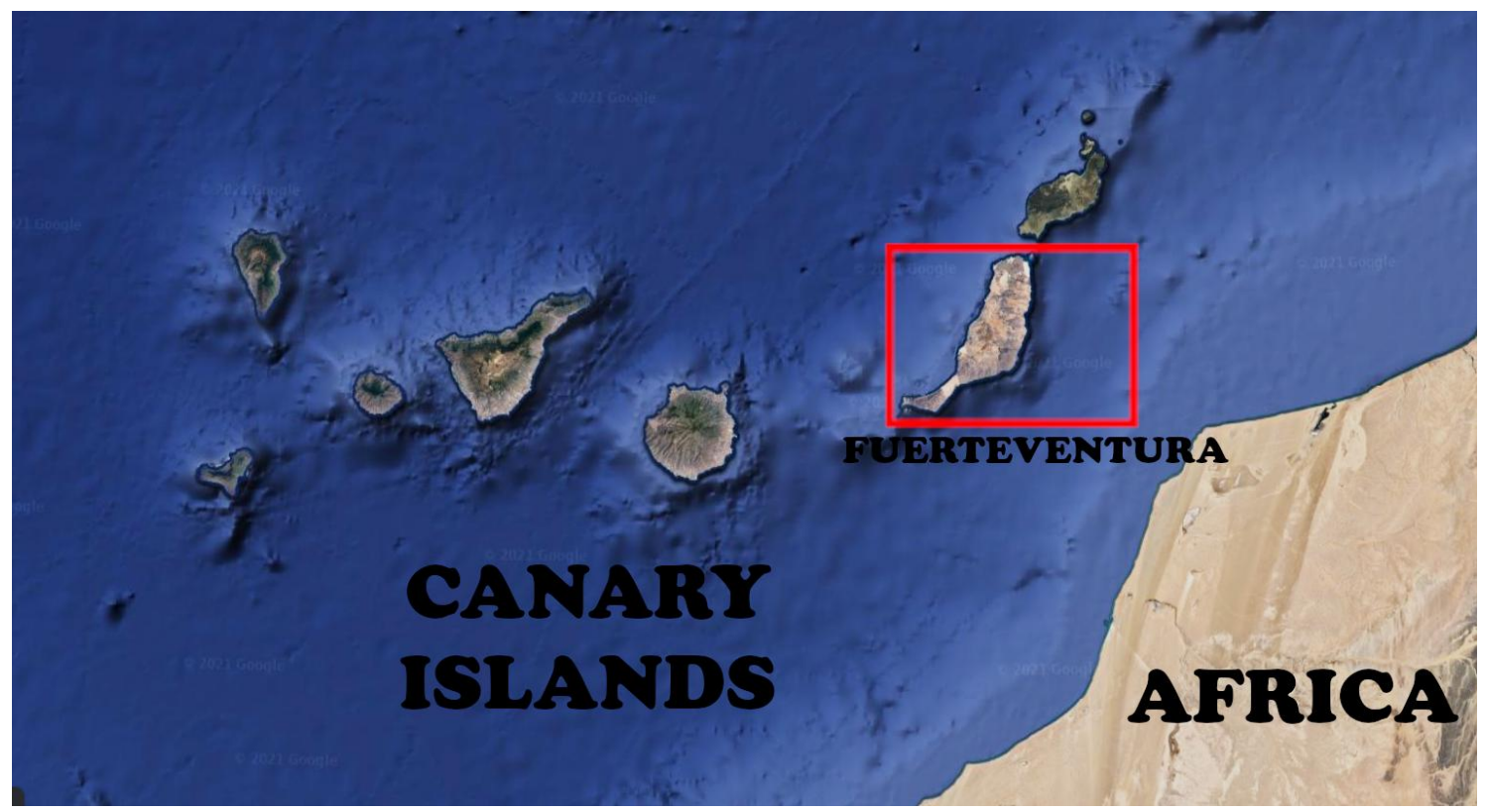

Canary Islands map.

Fuerteventura Island is the most ancient of them (23 million years old). It is only $90 \mathrm{~km}, 55.9$ miles, far from African coast. 
In addition, many other ancient rock lineal scripts are named Lineal Megalithic Writings or signs. Whether these signs represent one single or several languages, symbolic or utilitarian material (i.e.: measuring space/time/sky schemes) is not known. They have also been found in Iberia in a Megalithic context (thus its name): more or less unclassifiable lines and signs are found in Southern Spain like in Huelva and Cadiz dolmens (Vazquez-Hoys 2008; Arnaiz-Villena et al. 2013) respectively, and Antequera Dolmens ( Muñoz-Gambero 2019). Linear rocks incise lines and signs are also found in Northern Spain Pontevedra Stone, Galicia, Spain (Muñoz-Gambero 2019); some of them are mixed with typical Iberian semi-syllabary scripts, as the typical vocal "i" (Arnaiz-Villena, personal observation), (Muñoz-Gambero 2019; Arnaiz-Villena, Piedra de Pontevedra, Museo Galego, personal observation).

In summary, together with the so called Lineal Megalithic Scripts (VazquezHoys 2008; Muñoz-Gambero 2019) found in North and South Iberia, Iberian semisyllabary signs are found (Fig. 1). A continuum timeline of lineal painted and incised lines is found from lineal more ancient or Megalithic more recent alphabetic-like signs like Sitovo and Gradeshnitsa in Bulgaria (Fig. 2), which are dated older than 3,500 years BC, Iberian (Fig. 1) and syllabaries/alphabets evolution timeline may go back till about 70,000 years (in South Africa, Paleolithic) to 1st millennium BC and later.

\section{IBERIAN}

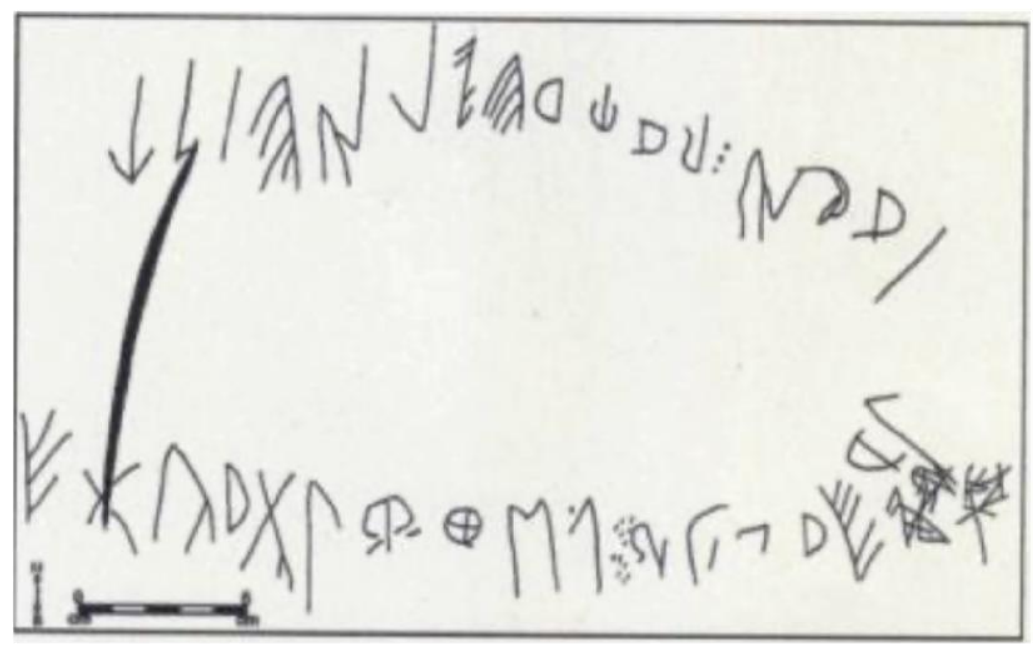

Fig. 1: Iberian incise rock inscriptions found in Cerdanya, Mt Pyrenees (Spain) and Lanzarote (Canary Islands, Spain) together with naviform likes which are a characteristic lineal rock Iberian Scripts. These naviform lines are also found in Canary Islands together with IberoGuanche rock scripts (Arnaiz-Villena et al. 2015; 2020b). 


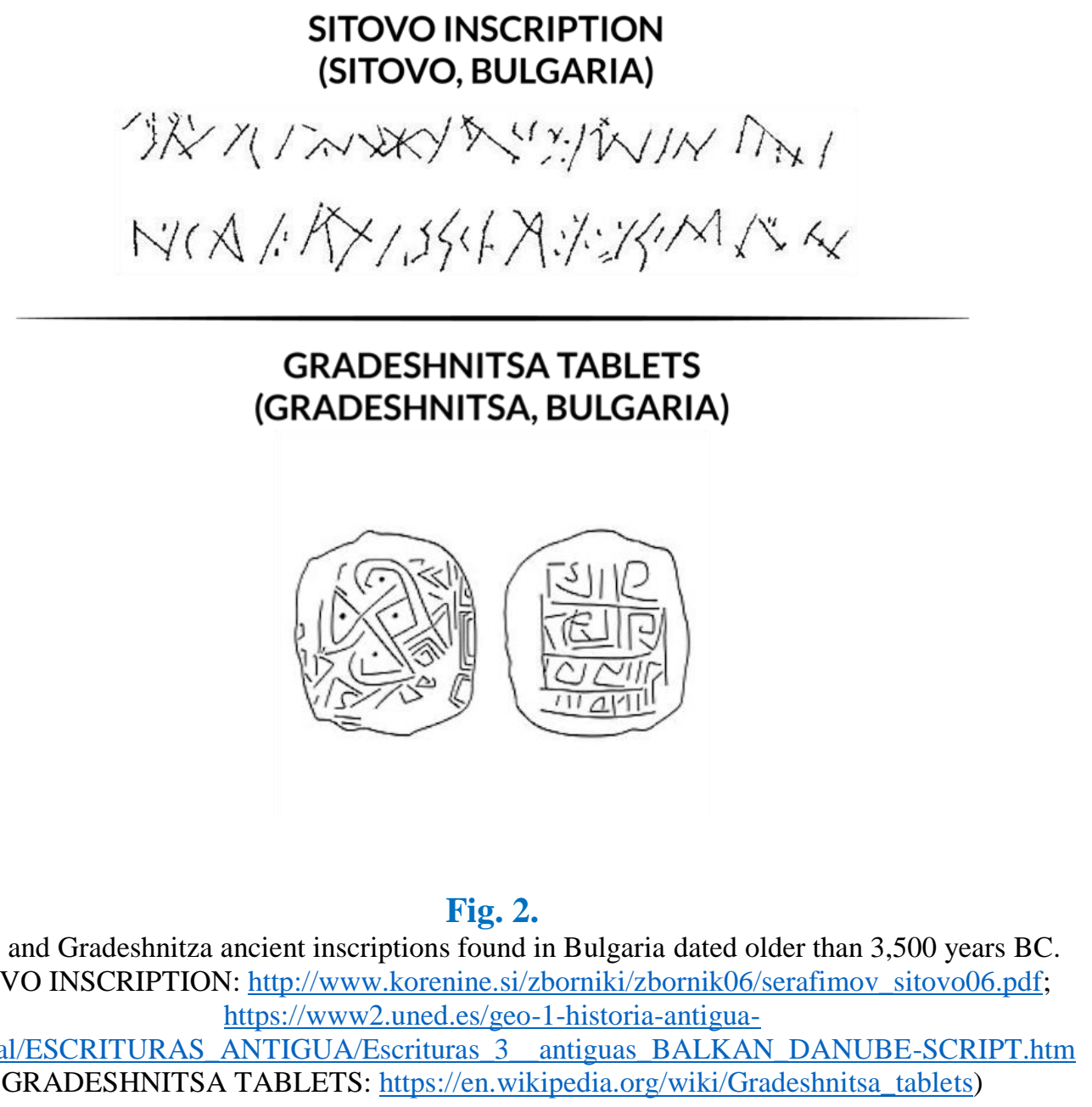

At some stages, incised lines without apparent syllable or letter representations are mixed together with classical recognizable syllables/letters, like naviform lines that go together in rocks with Iberian semi-syllabary both in Iberian and Canary Islands rocks (Arnaiz-Villena et al. 2015). It is likely that Iberian semi-syllabary existed long before than 1st millennium BC, because its antiquity has mainly been adapted to appear later than Phoenician alphabet and Phoenicians postulated arrival to Iberia and an urgent revision is required. Strabo wrote that Southwest Iberians had writing since 6,000 years BC (Strabo 1998). 


\section{Canarian prehistoric rock scripts}

We described the Iberian-Guanche rock Iberian writing twenty years ago found in Lanzarote and Fuerteventura (Arnaiz-Villena \& Alonso-García 2001). In Arnaiz-Villena et al. (2019b) we published that this type of rock writing was found in all Canary Islands and we named them Ibero-Guanche inscriptions because they could be transcripted and translated with Iberian semi-syllabary and postulated Basque language equivalences. Pichler named them "Latin" inscriptions (Pichler 2003). In addition, Canarian reputed scholars have also published lineal signs and/or incised or engraved lines in several papers/books that either have been hotly dismissed or neglected by other scholars like with our own findings have (Benito-Mateo et al. 2016; de BalbínBehrmann et al. 2009; del Arco-Aguilar et al. 2009; González-Antón et al. 1995; 2016). These Canarian authors and we may have been neglected because many of these findings do not fit with the strict genetic/cultural dogma present at Canary Islands which states that Canarian Anthropology is exclusively attached to Africa (Berbers) along all possible times. This dogma may logically be not possible and does not add any objective study to Canarian culture. It is particularly evident with the many small Canarian fat "goddesses" figurines (La Fortaleza, Ansite, Gran Canaria) and/or figurines which are similar to those found by Gimbutas in Europe $(6,000-3,000$ years BC) and also to Mediterranean Iberia figurines: scholars name this common traits with Canarian culture "The Gibraltar Strait Circle" that includes Mediterranean, Europe and Africa (González-Antón et al. 2016). We also add a similarity with singular Malta Archipelago culture: "fat goddesses", extant Cart-Ruts in both Canarian and Malta Archipelagos. Also pyramids are found in North Africa, Sahara Desert, Canary Islands and possibly southern Europe (Arnaiz-Villena et al. 2018; 2019c; 2020a; 2020b; 2020c; Medina and Arnaiz-Villena 2018a; 2018b). Some Iberian scholars have not studied Canary Islands Ibero-Guanche scripts because of "lack of enough material" (ArnaizVillena, personal talk with Iberian scholars); however, Italian lineal languages (Fig. 3) have been studied even with an extant few lines. Also, in Canary Islands not only Iberian scripts may be found but different stages of Lineal Megalithic Scripts or older Paleolithic (Vazquez-Hoys 2008; Muñoz-Gambero 2019) which may finally been reached full Iberian development in Canary Islands/Iberia and other parts of Europe 
with other lineal semi-syllabaries. A link between "Green" Sahara ancient rock script culture and Canarian Archipelago and also with African, European and Mediterranean lineal writing/scripts was lacking, and we present new evidence of such a relationship in the present paper. It is based on rock/stone epigraphy, since Iberian characters have been found in Central Sahara (Mt Ahaggar Area, South West Tamanrraset, Algeria and the new Lineal Megalithic scripts showed below in Fuerteventura Island (Figs 4, 5) as also showed in other Canary Islands.

\begin{tabular}{|c|c|c|c|c|c|}
\hline \multicolumn{2}{|c|}{ VENETIC } & \multicolumn{2}{|c|}{ LEPONTIC } & \multicolumn{2}{|c|}{ RAETIC } \\
\hline$A \cap A$ & & $\wedge A$ & & $A \wedge A \wedge$ & \\
\hline & 00 & & os & & 1 \\
\hline$>(=j)$ & 41 & & 1 & $>(?)$ & $M$ \\
\hline & $M r$ & & $\bowtie \bowtie$ & & 444 \\
\hline$\hat{\lambda}$ & 40 & $\hat{\lambda}$ & 4 & 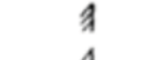 & $3 \xi$ \\
\hline$\lambda$ & $23 \xi$ & & $5 \xi$ & $\lambda$ & $x$ \\
\hline$\not x(=d)$ & $\times 1$ & $x$ & $x$ & $\not x \Psi_{(?)}$ & $x$ \\
\hline 日月小 & VA & & v & 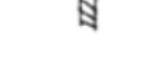 & $i \varphi \diamond$ \\
\hline$\odot_{(=t)}$ & $\diamond(=b)$ & $\odot$ & & 1 & \\
\hline $1_{x} \theta$ & & $\begin{array}{l}1 \\
x\end{array}$ & & $x$ & \\
\hline$J i$ & $Y(=g)$ & $\checkmark$ & & 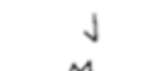 & $Y V$ \\
\hline $\begin{array}{l}M n \\
M n\end{array}$ & 日月怕的 & $\begin{array}{l}m i \\
4 n\end{array}$ & W & $\begin{array}{l}y \\
4\end{array}$ & \\
\hline
\end{tabular}

Fig. 3

Old Italian Lineal alphabets (not dated ).VENETIC, LEPONTIC,

RAETIC:https://en.wikipedia.org/wiki/Lepontic language\#/media/File:Venetic Raetic Camuni

c_Lepontic_alphabets.png

\section{Material and Methods}

A Sony Camera Cybershot 14.1 Megapixels Carl-Zeiss lens Vario-Tessar and Sony Xperia G3112 cellular phone camera were used for photograph work. 


\section{Results}

Examples of schematic lineal rock scripts from Africa, Europe and Mediterranean Area with a map of developed lineal writings placement.

Photographs of Lineal Megalithic/Paleolithic -like rock scripts at Fuerteventura

A difference should be point out between Paleolithic and Megalithic rock scripts. Megalithic ones are found in a Megalithic context even if in small stones far from Megaliths (not older than 7,000 years BP, see Arnaiz-Villena et al. 2015 and MuñozGambero 2019). Whether they represent syllables/letters of writing pointing out events, space/time measuring and /or symbols is not known. It is premature any kind of classification since the variety of scripts, their support and support size etc is overwhelming. They are found throughout Iberia and also recorded all over the World like South Africa (von Petzinger 2017; Henshilwood and D'Errico 2011, see Fig. 6). For example, Pichler (2003) thinks that Vinca script is borderline between structured writing and symbols and they are spread throughout Balcanic Peninsula and Romania.

The support of both Paleolithic and Megalithic lineal signs vary from small to bigger stones (intentionally polished or not) and cave or orthostate walls. In the schematic figures showed in the present paper, some examples of ancient languages lineal writing are shown from Africa, Mediterranean Area and Europe. In addition, Greek and Latin alphabets are also lineal. Latin and Geek were written in minor case only at Middle Age.

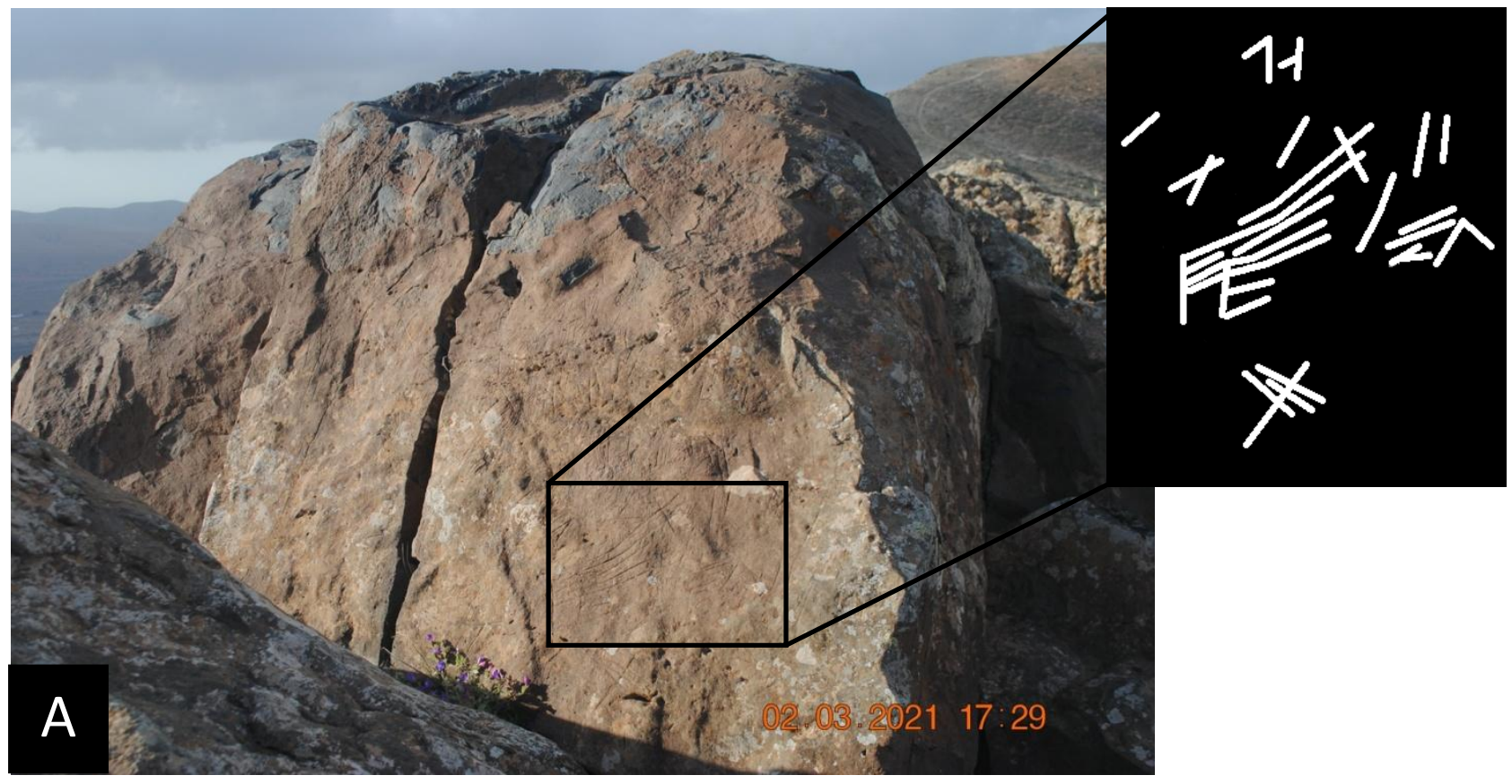



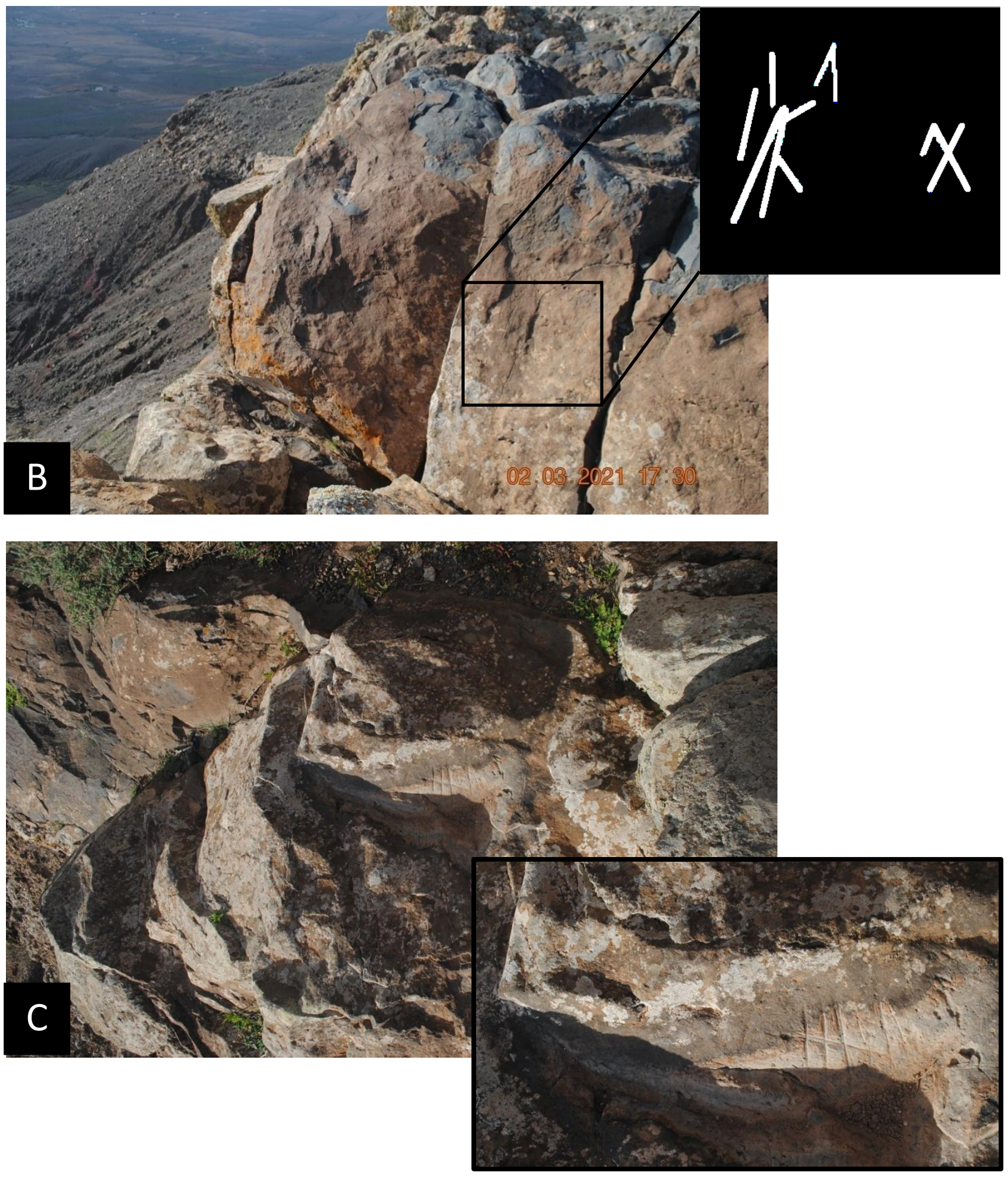

Fig. 4

A, B, C. Lineal Megalithic/Paleolithic-like rock scripts found in Los Castillejos (Ampuyenta), Fuerteventura (Canary Islands, Spain). Photos taken by authors. These type of inscriptions are found all around Fuerteventura both on rocks and small or bigger stones. Antiquity of inscriptions are not determined. 

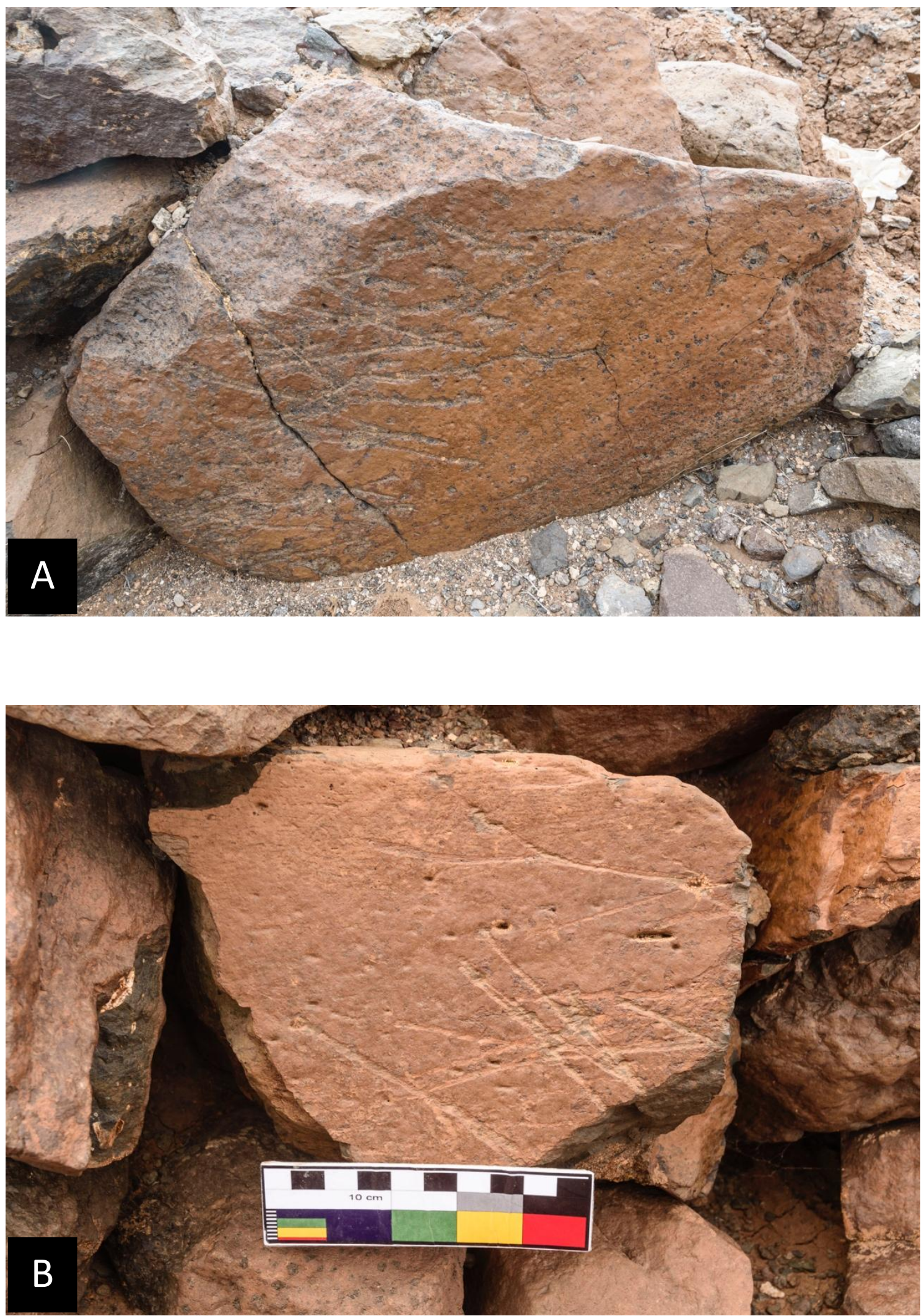

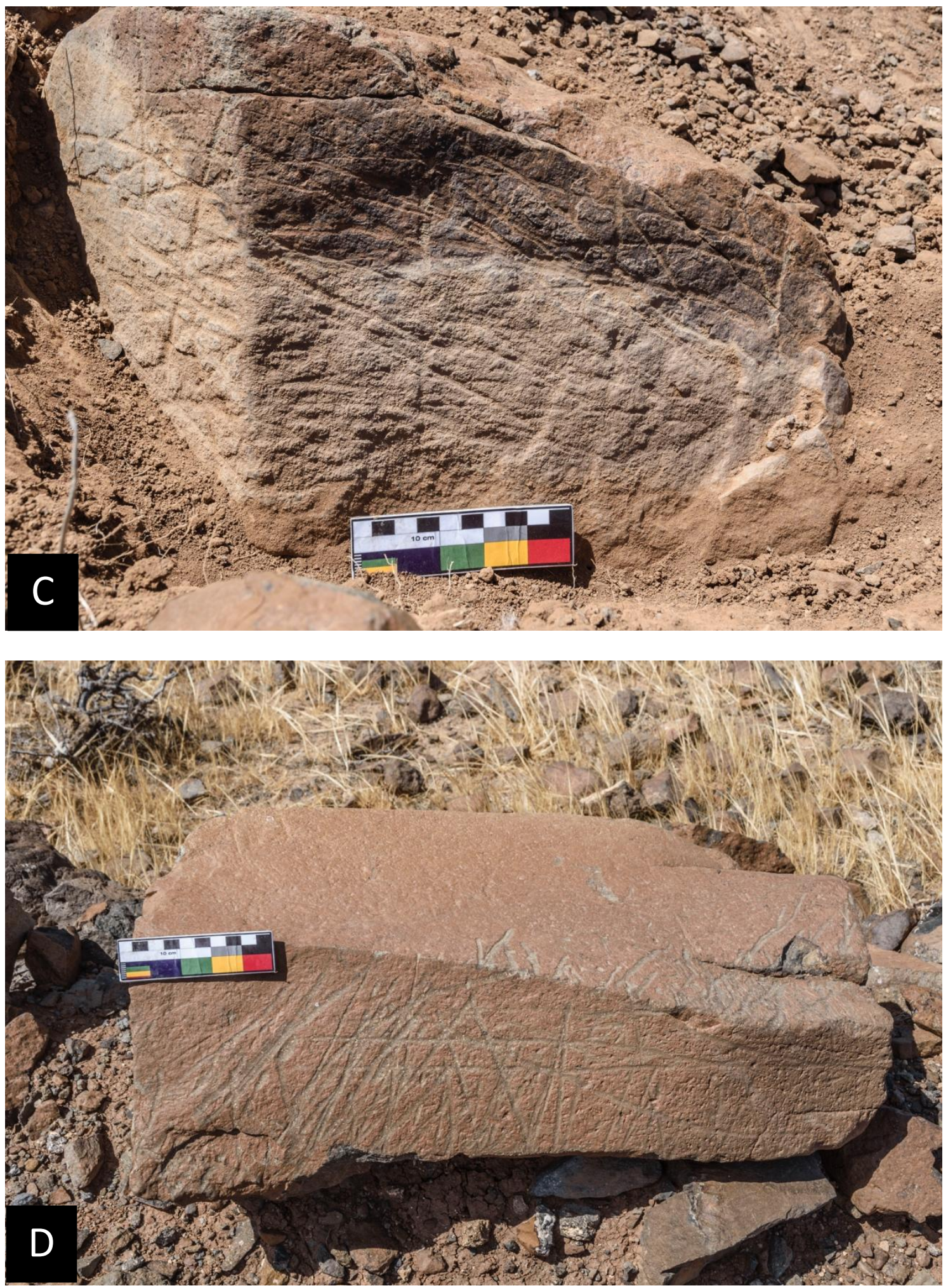

Fig. 5

A, B, C, D. Lineal Megalithic rock scripts found in Degollada de Facay, mountains that separate Tetir Valley from Tefia Valley, Fuerteventura (Canary Islands, Spain). Photos taken by authors. 


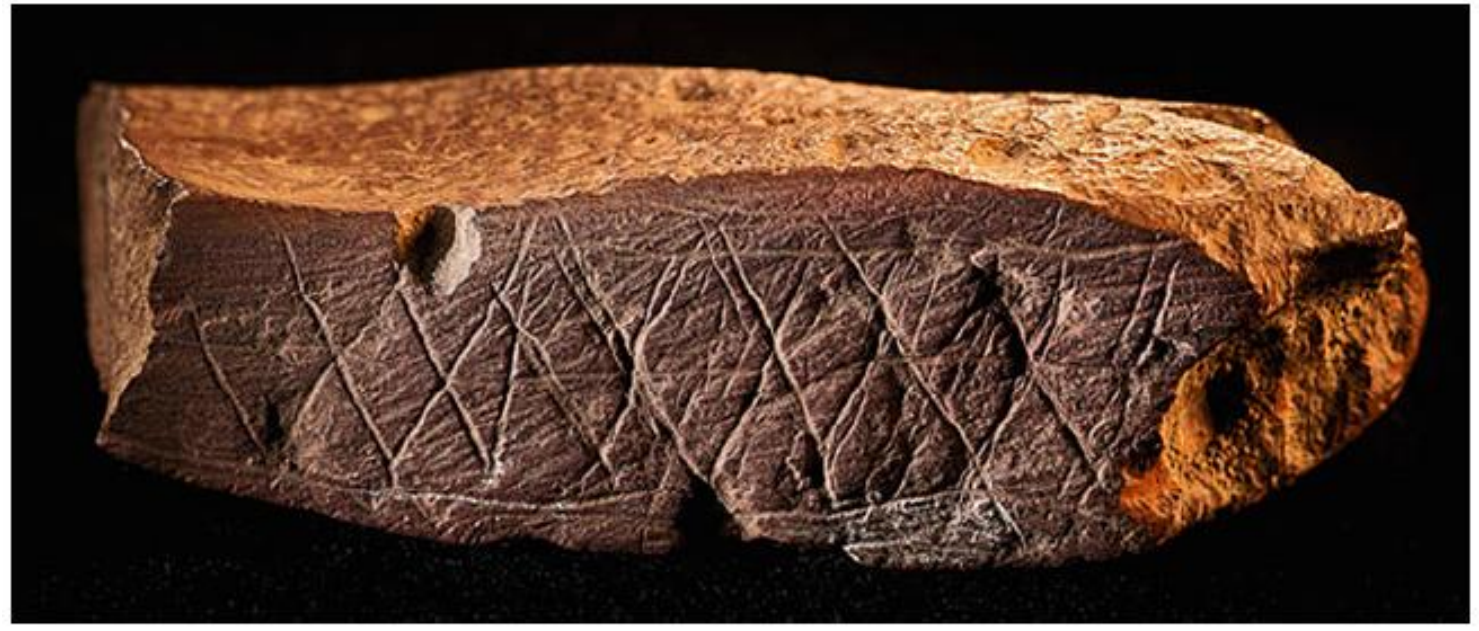

Fig. 6 Blombos Cave (South Africa). A lineal Paleolithic artifact with a regular incise design (70,000 years BP). It is dated long before than Lineal Megalithic scripts (Henshilwood \& D'Errico 2011). The represented incised motifs are very similar to those found at a Fuerteventura rock, which was donated to its Archaeological Museum on October 17th 2019 (Spanish EFE News Agency).

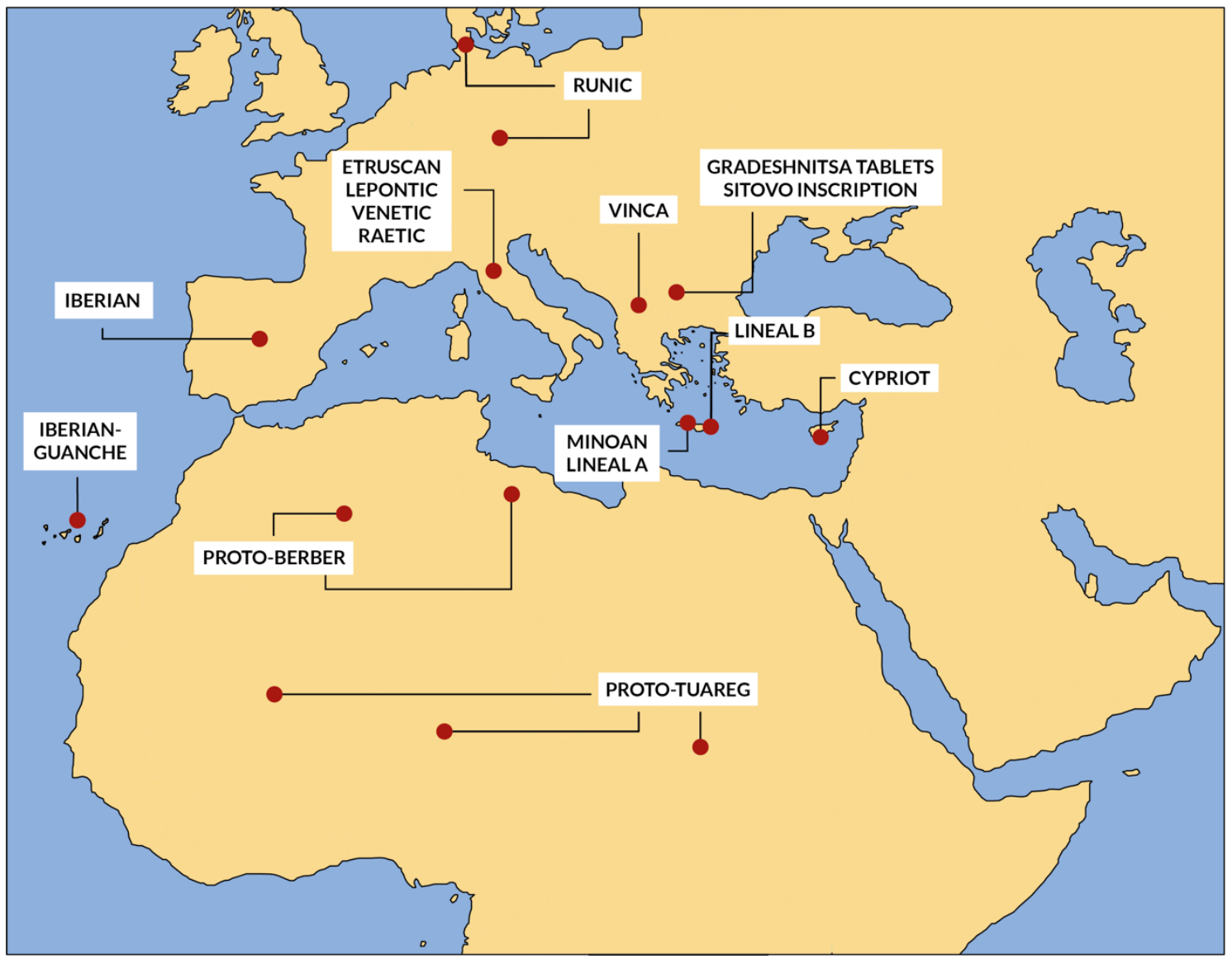

Fig. 7

Map showing examples of ancient Lineal Writings of Europe, Mediterranean Area, North Continental Africa and Canary Islands main locations. Also, Iberian phrases and signs have been found in the Middle of Sahara Desert, 200 km Southwest of Tamanrraset, Algeria (Arnaiz-Villena et al. 2021) 
PROTO-TUAREG

\begin{tabular}{|c|c|c|c|c|c|}
\hline thaggax & Air & & Ataggax & Air & \\
\hline Dா & (1) & $\mathbf{b}$ & ] & $J$ & $\mathbf{m}$ \\
\hline I. & 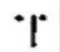 & $\mathbf{g}$ & 1 & I & $\mathbf{n}$ \\
\hline$\wedge \sqcap$ & & d & $\neq$ & & $\dot{\mathbf{n}}$ \\
\hline$\vdots$ & $\vdots$ & h & & & \\
\hline : & : & $\mathbf{w}$ & $\odot \square$ & $\odot$ & $\mathbf{s}$ \\
\hline ك & & $\check{\mathbf{z}}$ & $\vdots$ & $: \cdot:$ & $\gamma$ \\
\hline X & * & $\mathbf{z}$ & $\mathrm{X}$ & & g \\
\hline & $\longmapsto$ & c & $\cdots$ & $\vdots$ & $\mathbf{q}$ \\
\hline$\exists$ & $\exists$ & d & DO & 0 & $\mathbf{r}$ \\
\hline 23 & 4 & $\mathbf{y}$ & 608 & 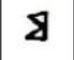 & $\check{\boldsymbol{s}}$ \\
\hline$\because:$ & $\because:$ & $\mathbf{k}$ & + & + & $t$ \\
\hline II & II & 1 & & & \\
\hline & & & • & $\bullet$ & , \\
\hline & & & $::$ & $::$ & $\mathbf{x}$ \\
\hline & & & 니 & 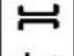 & $\mathbf{f}$ \\
\hline & & & \# & $\neq$ & \\
\hline
\end{tabular}

PROTO-BERBER

\begin{tabular}{|c|c|c|c|c|c|}
\hline III & H & 7\|\|$\|$ & T & $\mathbf{B C}$ & $\mathbf{S}$ \\
\hline$\odot$ & B & $N$ & I & $\mathbf{x}$ & $\mathbf{F}$ \\
\hline 1 & G & $1 \uparrow$ & $\mathbf{K}$ & $T$ & Ç \\
\hline$ᄃ$ & D & $=$ & $\mathbf{L}$ & $\div$ & $Q$ \\
\hline II & $\mathbf{U}$ & $\sqcup$ & $\mathbf{M}$ & 0 & $\mathbf{R}$ \\
\hline$\underline{\mathrm{m}} \mathrm{H}$ & Z Z & 1 & $\mathbf{N}$ & 3 & Ś \\
\hline$+\exists$ & $T T$ & & & & \\
\hline
\end{tabular}

Fig. 8

Transliterated Proto-Tuareg (Tifinagh) and Proto-Berber ancient linear alphabets. PROTO-

TUAREG: https://omniglot.com/writing/tifinagh.htm;

http://www.proel.org/index.php?pagina=alfabetos/tifinagh; PROTO-BERBER: Arnaiz-Villena \& Alonso-García 2001; Chabot 1940; 1941. 


\section{IBERIAN-GUANCHE}
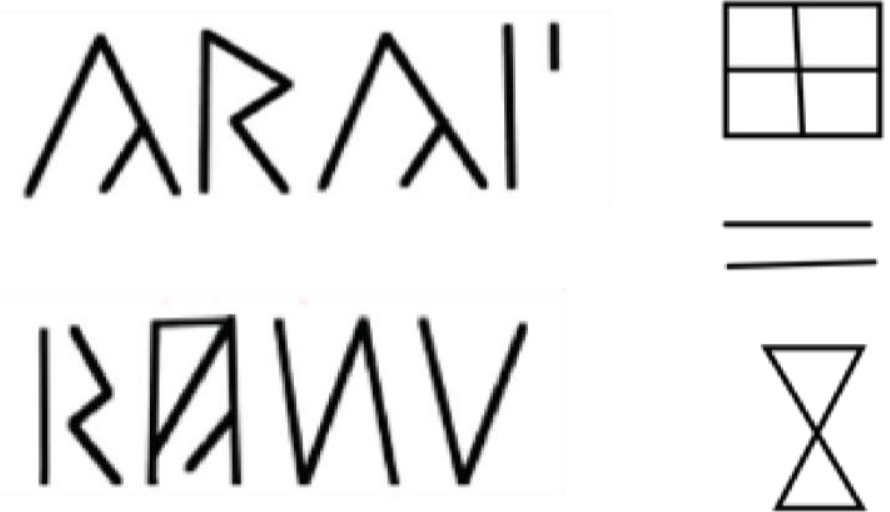

Fig. 9 Iberian-Guanche incise rock inscriptions found in Mt Tenezara,Lanzarote (Canary Islands, Spain) (Arnaiz-Villena et al. 2015; 2020b).

\section{ETRUSCAN}

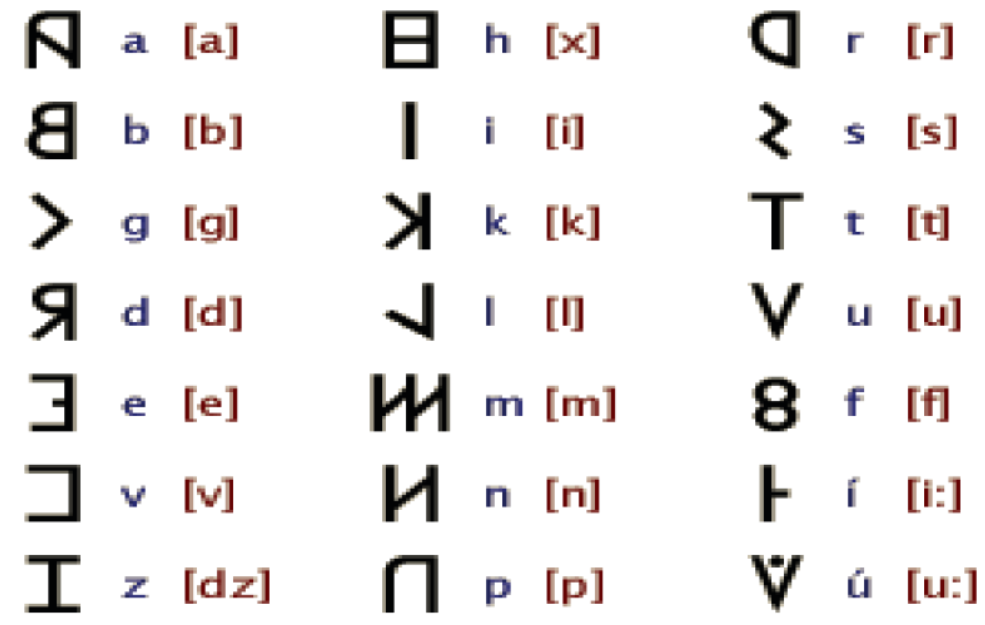

Fig. 10 ETRUSCAN: https://omniglot.com/writing/etruscan.htm; http://seansgallery.com/pages/h alphabets.htm 


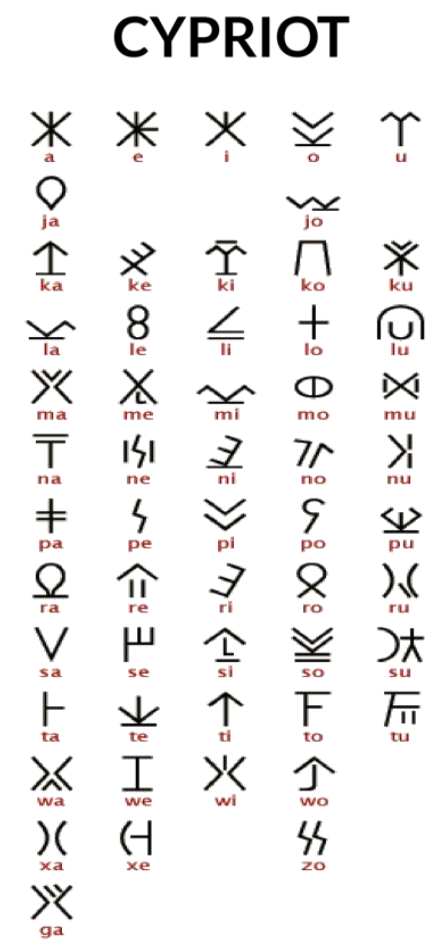

\section{MINOAN LINEAL A}

\begin{tabular}{|c|c|c|c|c|c|c|c|c|c|}
\hline & F & $\begin{array}{ll}A B & 9\end{array}$ & & & & & & $?$ & ${ }^{A B}$ \& \\
\hline$A B$ & + & $\begin{array}{ll}A B & A\end{array}$ & & C & & H & & $\oplus$ & ${ }^{A B}$ 雨 \\
\hline & & & $\frac{3 A}{A B}$ & & \begin{tabular}{|l|l}
55 \\
$A B$
\end{tabular} & & \begin{tabular}{|l|l|}
$\mathrm{AB}$ \\
\end{tabular} & & \\
\hline${ }_{03}^{A B}$ & $\neq$ & ${ }_{21^{m}}$ & & $\Lambda$ & & 目 & $\begin{array}{l}\mathrm{AB} \\
78\end{array}$ & () & ${ }_{1316}^{40}$ \\
\hline AB & 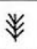 & $\begin{array}{ll}A B & \uparrow \\
22\end{array}$ & $A B$ & A & $\begin{array}{l}A B \\
57\end{array}$ & 目 & & vi & ${ }^{A}$ 䛔 \\
\hline & $\mp$ & $\begin{array}{ll}A B & \text { 等 }\end{array}$ & $A B$ & 屯 & & ᄃ & & 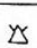 & ${ }^{A B}$ 区 \\
\hline & & $A B \cong$ & $A B$ & $A$ & $A B$ & Г & $A B$ & 3 & $A B \mp$ \\
\hline & & & & & & & & 2 & \\
\hline AB & $\mathrm{T}$ & AB \& & $A B$ & & $A B$ & $\mathrm{ls}$ & $A B$ & 13: & ${ }^{A B} \quad$ \\
\hline & & & $A B$ & & & & \begin{tabular}{|l|}
$A B$ \\
$A$
\end{tabular} & & \\
\hline & $T$ & $23^{m}=$ & & 米 & & 5 & 85 & $B$ & ${ }_{188}^{18}$ 日 \\
\hline $\begin{array}{l}A B \\
09\end{array}$ & $\mu$ & $\begin{array}{ll}A B & \text { IB } \\
{ }_{24} & \text {. }\end{array}$ & $\begin{array}{l}A B \\
C S\end{array}$ & in & $\begin{array}{l}A B \\
65\end{array}$ & in & $\begin{array}{l}A B \\
B 6\end{array}$ & 6 & $\begin{array}{ll}{ }^{A B} & Q\end{array}$ \\
\hline$A B$ & f & AB $\quad \psi$ & $A B$ & X & & 界 & & $\pi$ & ${ }^{A} \quad$ r \\
\hline$A B$ & & $A B \quad \psi$ & $A B$ & $\not$ & \begin{tabular}{|l|}
$A B$ \\
\end{tabular} & 18 & A & $\otimes$ & $A^{\prime} \Rightarrow$ \\
\hline 11 & 7 & ${ }_{27} T$ & 47 & & & & & 2 & \\
\hline$A B$ & $T$ & $\begin{array}{ll}A B & щ \\
28 & \end{array}$ & $A B$ & $\mathbb{I} \mathbb{I}$ & $A B$ & $\underset{\perp}{ \pm}$ & & d⿸丆口 & ${ }^{A} \leqslant$ \\
\hline & & & $A B$ & & & QP & & $\phi$ & A \\
\hline & I. & ${ }_{280} \psi^{*}$ & so & & & & & $T$ & 304 \\
\hline$A B$ & q & ${ }^{A B} \quad H$ & $\mathrm{AB}$ & 休 & $A B$ & $\forall$ & A & $\bar{\psi}$ & $A \sum$ \\
\hline & & $A B$ & & & & & & & $\frac{30 B}{A}$ \\
\hline & & & & & & & & & \\
\hline
\end{tabular}

\section{LINEAL B}

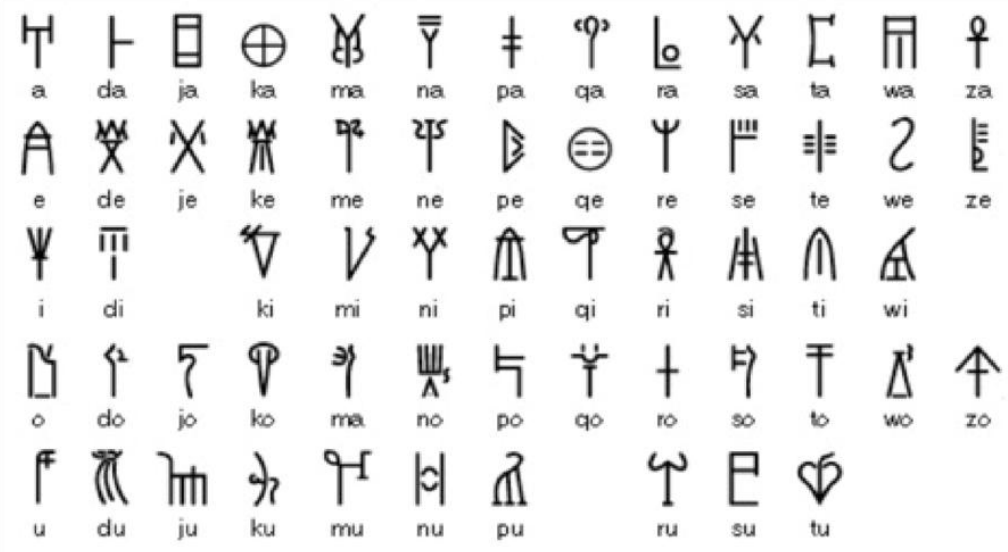

Fig. 11 Cypriot, Minoan Lineal A, and Lineal B ancient alphabets/syllabaries.

(CYPRIOT:https://omniglot.com/writing/cypriot.htm;

http://seansgallery.com/pages/h_alphabets.htm;

MINOAN LINEAL A: https://omniglot.com/writing/lineara.htm;

http://www.minoanatlantis.com/End_Minoan_Writing.php;

LINEAL B: http://blog.pompilos.org/archivo/to-no-to-e-re-ke-ro-re-a; Bernabé \& Luján 2007; 


\section{VINCA}

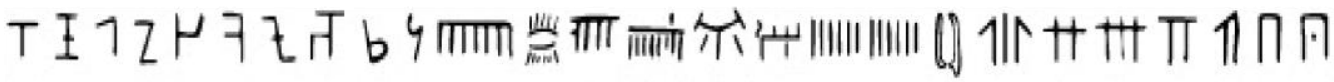

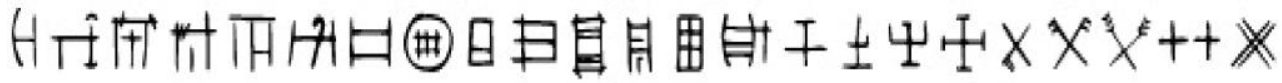

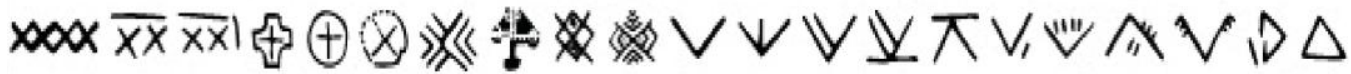

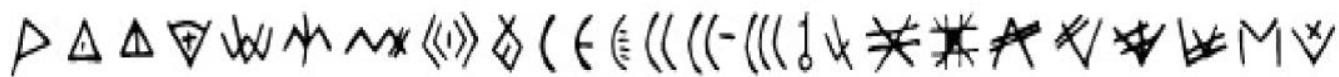

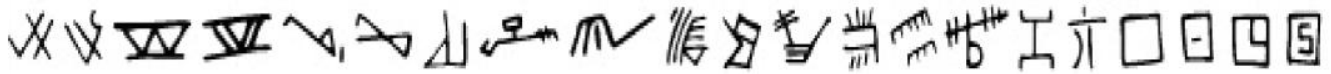

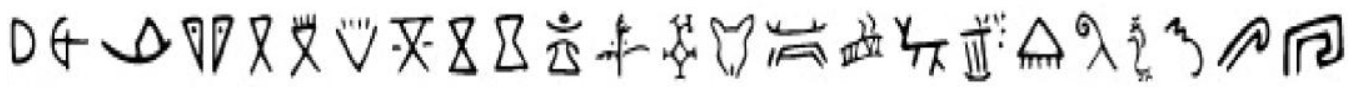

回它《纲

\begin{tabular}{|c|c|c|c|c|c|c|c|}
\hline \multicolumn{8}{|c|}{ RUNIC } \\
\hline P & $\bigcap$ & $D$ & $F$ & $\mathrm{R}$ & $<$ & $X$ & $P$ \\
\hline $\begin{array}{c}f \\
\text { fehu } \\
\text { wealth }\end{array}$ & $\begin{array}{c}\text { u } \\
\text { üruz } \\
\text { aurochs }\end{array}$ & $\begin{array}{c}p \\
\text { purisaz } \\
\text { giant }\end{array}$ & $\begin{array}{c}\text { a } \\
\text { ansuz } \\
\text { god }\end{array}$ & $\begin{array}{l}r \\
\text { raipō } \\
\text { riding }\end{array}$ & $\begin{array}{c}\mathrm{k} \\
\text { kaunaz } \\
\text { ulcer }\end{array}$ & $\begin{array}{c}\text { g } \\
\text { gebō } \\
\text { gift }\end{array}$ & $\begin{array}{c}\text { w } \\
\text { wunjōo } \\
\text { joy }\end{array}$ \\
\hline $\mathrm{H}$ & $t$ & 1 & ৬ & 5 & {[} & $\Psi$ & 4 \\
\hline $\begin{array}{c}\text { h } \\
\text { hagalaz } \\
\text { hail }\end{array}$ & $\begin{array}{c}n \\
\text { naupiz } \\
\text { need/hardship }\end{array}$ & $\begin{array}{l}\text { i } \\
\text { isa } \\
\text { ice }\end{array}$ & $\underset{\substack{\text { jera } \\
\text { year/harvest }}}{\mathrm{j}}$ & $\begin{array}{c}i \\
\text { eihwaz } \\
\text { yew tree }\end{array}$ & $\begin{array}{l}\mathrm{p} \\
\text { perp } \\
\text { luck }\end{array}$ & $\begin{array}{c}z \\
\text { algiz } \\
\text { sedge (?) }\end{array}$ & $\begin{array}{c}\text { s } \\
\text { sôwulō } \\
\text { sun }\end{array}$ \\
\hline$\uparrow$ & $B$ & $M$ & 円 & $\Gamma$ & $\diamond$ & W & $\hat{x}$ \\
\hline $\begin{array}{c}\mathrm{t} \\
\text { teiwaz } \\
\text { he god Tyr }\end{array}$ & $\begin{array}{c}\text { b } \\
\text { berkana } \\
\text { birch twig }\end{array}$ & $\begin{array}{c}\text { e } \\
\text { ehwaz } \\
\text { horse }\end{array}$ & $\begin{array}{c}\mathrm{m} \\
\operatorname{mannaz} \\
\operatorname{man}\end{array}$ & $\begin{array}{c}\text { I } \\
\text { laguz } \\
\text { water }\end{array}$ & $\begin{array}{c}\text { ng } \\
\text { inguz } \\
\text { the god Ing }\end{array}$ & $\begin{array}{c}\text { d } \\
\text { dagaz } \\
\text { day }\end{array}$ & $\begin{array}{c}\circ \\
\text { oppila } \\
\text { inherited lan }\end{array}$ \\
\hline
\end{tabular}

Fig. 12

Balkan Vinca and Scandinavian Runic signs. Runes are probably wrongly interpreted in this particular example. They are widespread throughout Scandinavia and Central Europe. Other authors interpreted them as letters /symbols.

VINCA: https://omniglot.com/writing/vinca.htm; https://www2.uned.es/geo-1-historia-antigua-

universal/ESCRITURAS ANTIGUA/Escrituras 3 antiguas BALKAN DANUBE-

SCRIPT.htm, font created by Sorin Paliga of the Department of Slavic Languages and Literature at the University of Bucharest, Romania;

RUNIC: https://omniglot.com/writing/runic.htm; https://en.wikipedia.org/wiki/Runes 


\section{Conclusions}

(See Figs 1-12)

1- Lineal Megalithic/Paleolithic signs/lines may have a variety of purposes or representations. Some authors have proposed: sky, planets, stars representations, space/time representations or others, including letters/syllables or symbols/events. Some are painted, other incised or picketed; the latter are relatively more common in Megalithic scripts.

2- Man is "writing" or creating handmade lineal figures on stones /rocks and other supports, which sometimes have intentionally been polished since Paleolithic times: at least 70,000 years BP (Blombos Cave, South Africa). Von Petzinger signs are over the World mainly in caves and are dated 40,000 years ago. Man had an early contact worldwide.

3- Megalithic script is named because it is associated to megalithic structures, although not exclusively. Von Petzinger "symbols" and/or writing is extended worldwide in Paleolithic caves and other rocks.

4- Canary Islands incise or picketed lineal writing exists mixed with transcribed and translated meaning signs (Ibero-Guanche or Latin inscriptions and Lybic ones). Other African/European/Mediterranean lineal scripts existed (see Fig. 7 map and Figs 1-12).

5- Fuerteventura Island contains in addition many small or bigger stones and rocks all over its territory. Timing in which these stones where incised by man is not known, we are only referring to a kind of stone crafting. However, we do not discard that they were made by man several thousand years ago.

6- Some Paleolithic/Megalithic scripts are mixed with clear Iberian semi-syllabary signs. They may reflect the evolution of more ancient Megalithic scripts to lineal writings like those detailed in Fig. 7 map and others.

7- Writing concept should be redefined whenever more precise data and dating be available. 


\section{Acknowledgements}

This work was supported by grants from the Spanish Ministry of Science and Universities PI18/00721 and European FEDER funds. We thank to Marco Antonio Blanco del Rio from Costa Calma (Fuerteventura) for being a continuous source of information.

Conflict of interest: The authors declare no conflict of interest.

\section{References}

Arnaiz-Villena A. \& Alonso-García J. 2001. Egipcios, Bereberes, Guanches y Vascos. Madrid, Spain: Ed. Visión Libros. (3rd Edition 2011). Amazon: https://www.amazon.es/Egipcios-Bereberes-Guanches-VascosLenguas/dp/841726700X; Wikimedia Commons: https://commons.wikimedia.org/wiki/File:Iberian-Guanche_inscriptions.pdf

Arnaiz-Villena A., Alonso-Rubio J., Ruiz-del-Valle V. 2013. Tiwanaku (Titikaka Lake, Bolivia) and Alberite Dolmen (Southern Spain) ritual "ears".Celtic, Iberian, Aymara and Basque languages. Int. J. Mod. Anthrop. 6: 61 - 76

Arnaiz-Villena A., Muñiz E ., Campos C ., Gómez-Casado E ., Tomasi S ., Martínez Quiles N., ...Palacio-Gruber J. 2015. Origin of Ancient Canary Islanders (Guanches): presence of Atlantic/Iberian HLA and Y chromosome genes and Ancient Iberian language. Int. J. Mod. Anthrop. 8: 67-93.

Arnaiz-Villena A., Medina M., Palacio-Gruber J., Lopez-Nares A., Ruiz-del-Valle V. 2018. Malta and Lanzarote (Canary Islands, Spain) Cart-ruts and Rock Prehistoric Calendar at Zonzamas, Lanzarote-“Quesera"/Cheeseboard.

Int. J. Mod. Anthrop. 11: 214-231.

Arnaiz-Villena A. Lopez-Nares A., Ruiz-del-Valle V. Juarez I., Bello A. and SanchezRomero G. 2019a. The Rock of the Dead: A New" Latin" or "Iberian-Guanche" Inscriptions found in Tenerife Is. (CanaryIslands, Spain).

Int. J. Mod. Anthrop. 2: 214- 232.

Arnaiz-Villena A., Lopez-Nares A., Juárez I., Ruiz-del-Valle V., Callado A., H-Sevilla A., Gomez-Casado E. 2019b. "Latín" rock scripts in Canary Islands are ancient Iberian inscriptions (Iberian-Guanche). A story of forgotten genetics, scripts, pyramids and other prehistoric artifacts. Int. J. Mod. Anthrop. 12: $189-212$. 
Arnaiz-Villena A., Medina M., Lopez-Nares A., Rodriguez-Rodriguez, J., Ruiz-del Valle V. 2019c. Cart-ruts in Lanzarote (Canary Islands, Spain) and Malta: first evidence of dating supported by dated ceramics. Int. J. Mod. Anthrop. 2: 115-140.

Arnaiz-Villena A., Suárez-Trujillo F., Ruiz-del-Valle V., López-Nares A., Pais-Pais F.J. 2020a. The Iberian-Guanche rock inscriptions at La Palma Is.: all seven Canary Islands (Spain) harbour these scripts. Int. J. Mod. Anthrop. 2 (14): 318 - 336.

Arnaiz-Villena A., Medina M., Ruiz-Del-Valle V., Lopez-Nares A., RodriguezRodriguez J., Suarez-Trujillo F. 2020b. The Ibero-Guanche (Latin) rock inscriptions found at Mt. Tenezara volcano (Lanzarote, Canary Islands, Spain): A Saharan hypothesis for Mediterranean/Atlantic Prehistory. Int. J. Mod. Anthrop. 2 (13): 140 162.

Arnaiz-Villena A., Medina M., Ruiz-del-Valle V., Lopez-Nares A., RodriguezRodriguez J., Suarez-Trujillo F. 2020c. Cart-ruts in Lanzarote (Canary Islands, Spain) volcanoes tops point to Equinoxes, Summer and Winter Solstices.

Int. J. Mod. Anthrop. 2(13): 123 - 138.

Arnaiz-Villena A, Ruiz-del-Valle V, López-Nares A, Suárez-Trujillo F. 2021. Iberian inscriptions in Sahara Desert rocks (Ti-m Missaou, Ahaggar Mts. area, Algeria) and first evidence of incise Iberian rock scripts in continental North Africa.

Int. J. Mod. Anthrop. 2(15): 440-467. DOI: http://dx.doi.org/10.4314/ijma.v2i15.3

Asociación sociocultural Archinife 2016. Chinech, la isla de los Letreros. Inscripciones de Piedra en Tenerife. Ed. Idea. Santa Cruz de Tenerife, Islas Canarias, España.

Benito-Mateo C., del Arco-Aguilar M.M., Rosario-Adrián M.C., González-Antón R ., del Arco-Aguilar C. 2016. Cerámicas antiguas en Rosita del Vicario (Fuerteventura, Islas Canarias). Una propuesta de trabajo. IN: XV Jornadas de estudios sobre Fuerteventura y Lanzarote. Puerto del Rosario, Spain: Cabildo de Fuerteventura.

Bernabé A \& Luján ER. 2007. Introducción al griego micénico. Gramática, selección de textos y glosario. Ed: Prensa de Universidad de Zaragoza. Zaragoza, Spain.

Chabot JB. 1940. Recueil des Inscriptions Libyques (fascicule premier). Ed: Imprimerie Nationale. Paris, France.

Chabot JB. 1941. Recueil des Inscriptions Libyques (fasciculesecond). Ed: Imprimerie Nationale. Paris, France.

De Balbín-Behrmann R., Bueno-Ramírez P., González-Antón R., del Arco-Aguilar C. 2009. Sea-land relationships in the rock art of the Prehispanic Canary Islands. IN: Grabados rupestres de la fachada atlántica europea y Africana . Rock Carvings of the European and African Atlantic Façade. Oxford, England: Arhcaeopress. 
Del Arco-Aguilar C., González-Antón R ., Rosario-Adrián M .C., del Arco-Aguilar M.M., González-Ginovés L., Benito-Mateo C., de Balbín-Behrmann, Bueno-Ramírez P. 2009. Algo má s que canalillos y geomé tricos. El valor simbó lico de las estaciones rupestres guanches. Canarias Arqueológica. 17: 79-131.

González-Antón R., de Balbín-Behrmann R., Bueno-Ramírez P., del Arco-Aguilar C. 1995. La piedra Zanata. Tenerife, Spain: Ed. OAMC, Cabildo de Tenerife.

González-Antón R., del Arco-Aguilar C., Rosario-Adrián M.C., Benito-Mateo C., del Arco-Aguilar M.M. 2016. Terracotas antropomorfas canarias antiguas . Una propuesta iconográfica. Datos para un poblamiento multiétnico . IN: XV Jornadas de estudios sobre Fuerteventura y Lanzarote. Puerto del Rosario, Spain: Cabildo de Fuerteventura.

Henshilwood C.S. \& Dubreuil B. 2011. The Still Bay and Howiesons Poort, 77-59 ka. Symbolic Material Culture and the Evolution of the Mind during the African Middle Stone Age. Current Anthropology. 3 (52): 361-400.

https://www.openculture.com/2019/03/40000-year-old-symbols-found-in-cavesworldwide-may-represent-the-earliest-written-language.html

Henshilwood C.S \& D’Errico F. 2011. Homo symbolicus: the dawn of language, imagination and spirituality. Ed. John Benjamins Pub. Co. Amsterdam, Holand.

Kober AE. 1948. The Minoan Scripts: Fact and Theory. American Journal of Archaeology 52 (1): 82-103.

Medina M., Arnaiz-Villena A. 2018a. A Lunisolar Prehistoric Calendar in Lanzarote Island: "La Quesera" (Cheeseboard) from Zonzamas. In. J. Mod. Anthrop. 2: 147-161.

Medina M., Arnaiz-Villena A. 2018b. The Moon: in Prehistoric Lunisolar Rock Calendar "Quesera"- Cheeseboard- at Lanzarote, Canary Islands, Spain.

Int. J. Mod. Anthrop. 2: 182-212.

Muñoz-Gambero J.M. 2019. El origen de la escritura . La magia de los símbolos . Ed. Fundación Unicaja. Málaga, Spain.

Pichler W. 2003. Las inscripciones rupestres de Fuerteventura. Ed. Cabildo de Fuerteventura. Puerto del Rosario, Spain.

Strabo 1998. Geografía. Book III, Part 6, lastparagraph. Ed. Biblioteca Clásica Gredos . Madrid, Spain.

Texier P.J., Porraz G., Parkington J., Rigaud J.P., Poggenpoel C., Miller C., ...Verna C. 2010. A Howiesons Poort tradition of engraving ostrich eggshell containers dated to 60,000 years ago at Diepklo of Rock Shelter, South Africa. PNAS. 107(14): 6180-6185.

Vázquez-Hoys A.M. 2008. Las golondrinas de Tartesos: sobre el origen de la escritura. Ed. Almuzara. Córdoba, España 
Von Petzinger G. 2017. The first signs: unlocking the mysteries of the world's oldestsymbols. New York, USA: Atria

Paperback. https://www.openculture.com/2019/03/40000-year-old-symbols-found-incaves-worldwide-may-represent-the-earliest-written-language.html

To cite this article:

Arnaiz-Villena A., Medina M., Ruíz-del-Valle V., López-Nares A., de Vera-Lima J.A., Mata L., Barrera-Gutiérrez L., Palacio-Grüber J., Suarez-Trujillo F. 2021. Lineal Megalithic Rock Scripts as precursors of Iberian and other lineal Mediterranean/Euro African ancient writings: the case of Fuerteventura (Canary Islands, Spain).

International Journal of Modern Anthropology. 2 (16): 629 - 648

DOI: http://dx.doi.org/10.4314/ijma.v2i16.6

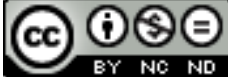

This article, as all articles published in this journal, is under The Creative Commons Attribution: Attribution-NonCommercial-NoDerivatives 4.0 International (CC BY-NC-ND 4.0). https://creativecommons.org/licenses/by-nc-nd/4.0/ 